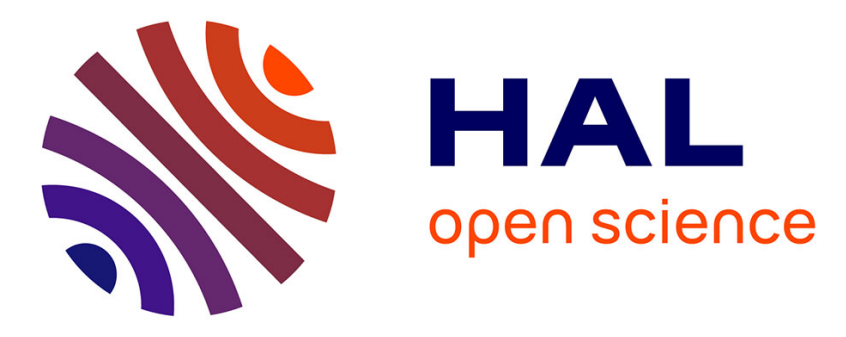

\title{
Temperature-driven massless Kane fermions in HgCdTe crystals
}

Frederic Teppe, M. Marcinkiewicz, Sergey S. Krishtopenko, Sandra Ruffenach, Christophe Consejo, Aleksandr Kadykov, Wilfried Desrat, Dmytro But, Wojciech Knap, J. Ludwig, et al.

\section{To cite this version:}

Frederic Teppe, M. Marcinkiewicz, Sergey S. Krishtopenko, Sandra Ruffenach, Christophe Consejo, et al.. Temperature-driven massless Kane fermions in $\mathrm{HgCdTe}$ crystals. Nature Communications, 2016, 7, pp.12576. 10.1038/ncomms12576 . hal-01391282

\section{HAL Id: hal-01391282 \\ https://hal.science/hal-01391282}

Submitted on 9 Jun 2021

HAL is a multi-disciplinary open access archive for the deposit and dissemination of scientific research documents, whether they are published or not. The documents may come from teaching and research institutions in France or abroad, or from public or private research centers.
L'archive ouverte pluridisciplinaire HAL, est destinée au dépôt et à la diffusion de documents scientifiques de niveau recherche, publiés ou non, émanant des établissements d'enseignement et de recherche français ou étrangers, des laboratoires publics ou privés.

\section{(c)(1)}

Distributed under a Creative Commons Attribution| 4.0 International License 


\section{ARTICLE}

\section{Temperature-driven massless Kane fermions in $\mathrm{HgCdTe}$ crystals}

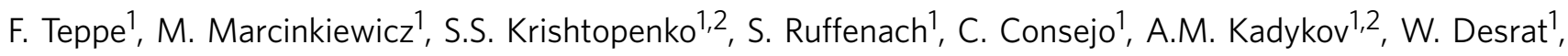
D. But 1 , W. Knap 1,3, J. Ludwig4,5, S. Moon 4,5, D. Smirnov4, M. Orlita6,7, Z. Jiang8, S.V. Morozov 2,9, V.I. Gavrilenko ${ }^{2,9}$, N.N. Mikhailov ${ }^{10,11} \&$ S.A. Dvoretskii ${ }^{10,11}$

It has recently been shown that electronic states in bulk gapless $\mathrm{HgCdTe}$ offer another realization of pseudo-relativistic three-dimensional particles in condensed matter systems. These single valley relativistic states, massless Kane fermions, cannot be described by any other relativistic particles. Furthermore, the $\mathrm{HgCdTe}$ band structure can be continuously tailored by modifying cadmium content or temperature. At critical concentration or temperature, the bandgap collapses as the system undergoes a semimetal-to-semiconductor topological phase transition between the inverted and normal alignments. Here, using far-infrared magneto-spectroscopy we explore the continuous evolution of band structure of bulk $\mathrm{HgCdTe}$ as temperature is tuned across the topological phase transition. We demonstrate that the rest mass of Kane fermions changes sign at critical temperature, whereas their velocity remains constant. The velocity universal value of $(1.07 \pm 0.05) \times 10^{6} \mathrm{~ms}^{-1}$ remains valid in a broad range of temperatures and $\mathrm{Cd}$ concentrations, indicating a striking universality of the pseudo-relativistic description of the Kane fermions in $\mathrm{HgCdTe}$.

\footnotetext{
${ }^{1}$ Laboratoire Charles Coulomb, UMR CNRS 5221, University of Montpellier, Montpellier 34095, France. ${ }^{2}$ Institute for Physics of Microstructures, Russian Academy of Sciences, Nizhny, 603950 GSP-105 Novgorod, Russia. ${ }^{3}$ Institute of High Pressure Institute Physics, Polish Academy of Sciences, 01-447 Warsaw, Poland. ${ }^{4}$ National High Magnetic Field Laboratory, Tallahassee, Florida 32310, USA. ${ }^{5}$ Department of Physics, Florida State University, Tallahassee, Florida 32306 , USA. ${ }^{6}$ Laboratoire National des Champs Magnétiques Intenses, CNRS-UJF-UPS-INSA, 38042 Grenoble, France. ${ }^{7}$ Faculty of Mathematics and Physics, Charles University, Ke Karlovu 5, 12116 Prague 2, Czech Republic. ${ }^{8}$ School of Physics, Georgia Institute of Technology, Atlanta, Georgia 30332, USA. ${ }^{9}$ Lobachevsky State University of Nizhny Novgorod, Nizhny, 603950 Novgorod, Russia. ${ }^{10}$ Institute of Semiconductor Physics, Siberian Branch, Russian Academy of Sciences, pr. Akademika Lavrent'eva 13, 630090 Novosibirsk, Russia. ${ }^{11}$ Novosibirsk State University, 630090 Novosibirsk, Russia. Correspondence and requests for materials should be addressed to F.T. (email: Frederic.Teppe@umontpellier.fr).
} 
$\mathrm{n}$ condensed matter systems, the interaction of electrons with a periodic crystal lattice potential can give rise to low-energy quasiparticles that mimic the relativistic dynamics of Dirac particles in high-energy physics. Perhaps the most spectacular demonstration of this concept was given ten years ago by the isolation of a monolayer of carbon atoms forming a graphene ${ }^{1}$ sheet. The electrons in graphene behave as two-dimensional (2D) massless fermions with gapless conical bands that obey the Dirac equation. Subsequently, further condensed matter analogues of high-energy relativistic fermions were demonstrated such as edge or surface states of $2 \mathrm{D}$ or three-dimesnional (3D) topological insulators $^{2-4}$ and 3D Dirac semimetals with linear energymomentum dispersion in all three momentum directions ${ }^{5-7}$.

Recently, another massless Dirac-like quasiparticle has been discovered in $\mathrm{Hg}_{1-x} \mathrm{Cd}_{x} \mathrm{Te}$ at an inverted-to-normal band structure topological transition existing at the critical cadmium concentration ${ }^{8} x_{\mathrm{C}} \approx 0.17$. These $3 \mathrm{D}$ massless Kane fermions are not equivalent to any other known relativistic particles. Similar to the pseudospin-1 Dirac-Weyl system ${ }^{9}$, their energy dispersion relation features cones crossed at the vertex by an additional flat band. The bandgap and the electronic dispersion in $\mathrm{Hg}_{1-x} \mathrm{Cd}_{x} \mathrm{Te}$ can be tuned intrinsically by adjusting the chemical composition, or externally, by changing temperature ${ }^{10}$. The ability to control the properties of quasiparticles with relativistic behaviour in a tabletop condensed-matter experiment holds vast scientific and technological potential. However, the variation of the chemical composition in $\mathrm{Hg}_{1-x} \mathrm{Cd}_{x} \mathrm{Te}$ crystals does not allow for fine-tuning of the bandgap in the vicinity of the phase transition due to inherent fluctuations of Cd concentration. Contrarily, a temperature-driven evolution of the band structure (see also Supplementary Note 1) provides a conceptually straightforward, yet very accurate and detailed probe of the relativistic properties of Kane fermions, while tuning the $3 \mathrm{D}$ $\mathrm{Hg}_{1-x} \mathrm{Cd}_{x} \mathrm{Te}$ across the gapless state at the topological transition between a normal state and an inverted bandgap state. The appearance of a non-zero gap does not exclude relativistic properties of the Kane fermions in $\mathrm{Hg}_{1-x} \mathrm{Cd}_{x} \mathrm{Te}$, which is retained at energies significantly above the gap. The energy dispersion asymptotically tends to a linear behaviour, as it is for relativistic electrons at high energies. The cutoff energies for relativistic behaviour in the $\mathrm{Hg}_{1-x} \mathrm{Cd}_{x} \mathrm{Te}$ compounds and in other 3D Dirac-Weyl semimetals are related to the presence of high-lying conduction bands and low-lying valence bands.

Here we conduct a systematic optical investigation of the dispersion of Kane fermions in $\mathrm{Hg}_{1-}{ }_{x} \mathrm{Cd}_{x} \mathrm{Te}$ crystal as a function of temperature and magnetic field. From the experimental point of view, the use of temperature as a band structure tuning parameter in magneto-optical studies is challenging and demands ultimate quality samples. This is because the observation of welldefined optical resonances requires high carrier mobility, which degrades with increasing temperature due to the increase of scattering on phonons. Our bulk $\mathrm{Hg}_{1-x} \mathrm{Cd}_{x} \mathrm{Te}$ samples were grown by molecular beam epitaxy. The $\mathrm{Cd}$ concentration was chosen to enable exploring the bandgap $E_{\mathrm{g}}(x, T)$ space across the semimetal-to-semiconductor phase transition using temperature for fine gap-at-will tuning. The sample A, $x=0.175$, is a standard narrow-gap semiconductor at any temperature. The sample $B$, $x=0.155$, is a semimetal at low temperatures with a negative bandgap corresponding to the inverted band order. As the temperature increases, the inverted bandgap closes as the system undergoes a semimetal-to-semiconductor phase transition at the critical temperature $T_{\mathrm{C}} \approx 77 \mathrm{~K}$ followed by the opening of a gap in the normal state. Based on a simplified Kane model, we determine the Kane fermions velocity and rest mass. The rest mass experiences a change of sign at the critical temperature of topological phase transition. Our study reveals a universal velocity of $1.07 \times 10^{6} \mathrm{~m} \mathrm{~s}^{-1}$ in HgTe crystals. This further allows to determine the Kane fermion rest mass from all experimental results, past or future, close to the phase transition.

\section{Results}

Band structure evolution with temperature. To describe the electronic structure near the centre of the Brillouin zone in $\mathrm{Hg}_{1-{ }_{x}} \mathrm{Cd}_{x} \mathrm{Te}$ close to $x_{\mathrm{C}}$, we employ a simplified Kane model ${ }^{11,12}$ taking into account $k \cdot p$ interaction between the $\Gamma_{6}$ and $\Gamma_{8}$ bands, while neglecting the influence of the split-off $\Gamma_{7}$ band. The corresponding $(6 \times 6)$ Hamiltonian formally resembles the one for relativistic 3D Dirac particles (see 'Simplified Kane model' in Methods). By neglecting small quadratic in momentum terms, the eigenvalues of this Hamiltonian can be presented in the form:

$$
\begin{aligned}
E_{\xi=0}(p) & =0, \\
E_{\xi}(p) & =\xi^{2} \tilde{m} \tilde{c}^{2}+(-1)^{1-\theta(\tilde{m})} \xi \sqrt{\tilde{m}^{2} \tilde{c}^{4}+p^{2} \tilde{c}^{2}},
\end{aligned}
$$

The first eigenvalue $\xi=0$ corresponds to the flat heavy-hole band. The two other eigenvalues describe the electron $(\xi=+1)$ and light-hole $(\xi=-1)$ conical bands separated by $E_{\mathrm{g}}=2 \tilde{m} \tilde{c}^{2}$. $\theta(\tilde{m})$ is a Heaviside step function, which equals to 1 for $\tilde{m} \geq 0$ and to 0 if $\tilde{m}$ is negative. This representation of Kane fermions in $\mathrm{Hg}_{1-x} \mathrm{Cd}_{x} \mathrm{Te}$ contains only two parameters, the rest -mass $\tilde{m}$ and the universal velocity $\tilde{c}=\sqrt{2 P^{2} /\left(3 \hbar^{2}\right)}$, whereas the material properties are introduced through the Kane's matrix element $P$ and the $\mathrm{Hg}_{1-x} \mathrm{Cd}_{x}$ Te bandgap $E_{\mathrm{g}}$.

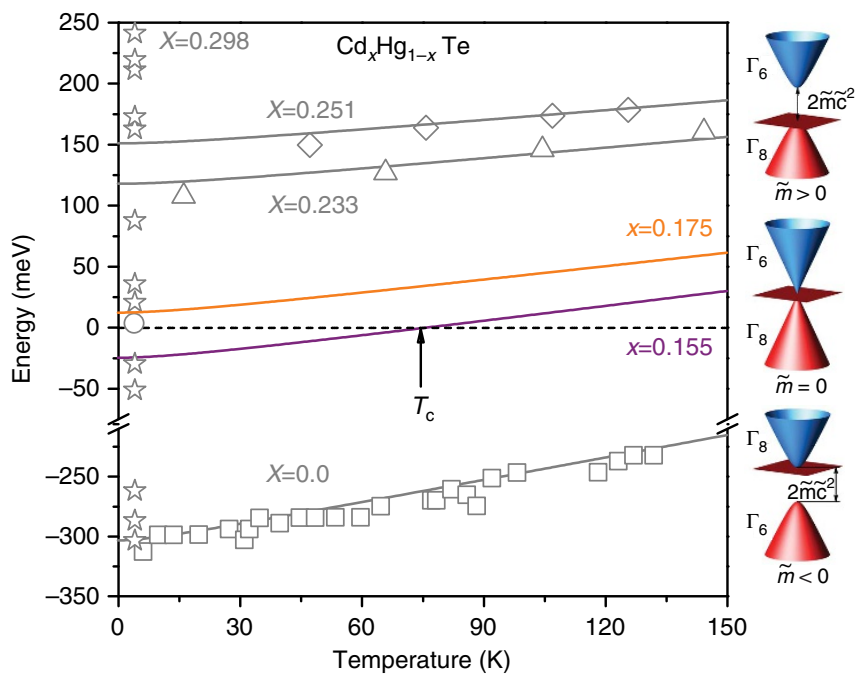

Figure 1 | Temperature tuning of the band structure in $3 \mathrm{D} \mathbf{H g}_{x} \mathbf{C d}_{\mathbf{1}_{\mathbf{x}}} \mathbf{T e}$. (a) The energy bandgap in $\mathrm{Hg}_{x} \mathrm{Cd}_{1-x} \mathrm{Te}$, defined as the difference between the $\Gamma_{6}$ and $\Gamma_{8}$ band extrema at the centre of the Brillouin zone, increases monotonically with temperature and $\mathrm{Cd}$ content. Open symbols correspond to experimental data, with circle from ref. 8, squares from ref. 21 , stars from ref. 23, and triangles and diamonds from ref. 24, for several $\mathrm{Cd}$ concentrations, and the lines show the $E_{\mathrm{g}}(T)$ evolution calculated using Supplementary equation (1) ${ }^{21}$ (in Supplementary Note 1). The purple and orange lines correspond to the $\mathrm{Cd}$ concentration for samples studied in this work, $x=0.155$ and $x=0.175$. At the critical temperature, $T_{C}$, the system undergoes a semimetal-to-semiconductor topological phase transition between inverted and normal states. For $x=0.155$, the gapless state is realized at $T_{\mathrm{C}} \approx 77 \mathrm{~K}$. (b) Schematic band structure of Kane fermions in $3 \mathrm{D} \mathrm{Hg} \mathrm{Cd}_{1-x} \mathrm{Te}$. As the rest mass of Kane Fermions changes, the electronic dispersion evolves from a standard gapped semiconductor for $\tilde{m}>0$ into a semimetal at $\tilde{m}<0$. At the point of the topological transition, $\tilde{m}=0$, the conical conduction (blue) and the light-hole valence (red) bands are crossed at the vertex by a flat heavy-hole band (brown). 
The evolution of Kane fermions in $\mathrm{Hg}_{1-{ }_{x}} \mathrm{Cd}_{x} \mathrm{Te}$ is illustrated in Fig. 1. If the rest mass $\tilde{m}$ is positive, the crystal is a typical narrow-gap semiconductor with the $s$-type $\Gamma_{6}$ band lying above the $p$-type $\Gamma_{8}$ bands, as schematically shown on Fig. 1a. On the other hand, if $\tilde{m}<0$, the band order is inverted: the $\Gamma_{6}$ band lies below the $\Gamma_{8}$ bands. As the two $\Gamma_{8}$ bands always touch each other at the $\Gamma$ point of the Brillouin zone, the band structure is gapless and the crystal is a semimetal. The two distinct phases with different sign of the rest mass are not topologically equivalent, as characterized by a $Z_{2}$ topological invariant ${ }^{13}$.

Experimentally, the dispersion of gapless or gapped $2 \mathrm{D}$ or $3 \mathrm{D}$ Dirac fermions can be conveniently probed through the magnetic field dependence of inter-Landau level transitions ${ }^{14-20}$. The application of a quantizing magnetic field $B$ transforms the zero-field continuum of states into a set of unequally spaced Landau levels (LLs) with a distinct $\sqrt{B}$ behaviour. In pristine gapless graphene, for example, the LLs have a simple structure given by ${ }^{14}: E_{n}=\operatorname{sgn}(n) \sqrt{2|n| e \hbar \tilde{c}^{2} B}$, where $\hbar$ is the Planck constant and $e$ is the electron charge. The integer LL index $n$ labels electron- $(n>0)$ and hole-like $(n<0)$ states, and unconventional, zero-energy field-independent $n=0 \mathrm{LL}$ states. In graphene/boron-nitride heterostructures with zero crystallographic alignment angle, an intrinsic gap $\Delta$ opens up separating zeroth dispersionless LLs, $E_{n=0}= \pm \Delta / 2$. Other $|n|>0$ electron(hole)-like LLs shift up(down) by $\Delta / 2$ as well: $E_{|n|>0}=\operatorname{sgn}(n) \sqrt{2|n| e \hbar \tilde{c}^{2} B+(\Delta / 2)^{2}}$ (after ref. 17).

The LL spectrum of massive or massless fermions in $\mathrm{Hg}_{1-x} \mathrm{Cd}_{x} \mathrm{Te}$ has a more complex form:

$$
E_{\xi, n, \sigma}\left(p_{z}\right)=\xi^{2} \tilde{m} \tilde{c}^{2}+(-1)^{1-\theta(\tilde{m})} \xi \sqrt{\tilde{m}^{2} \tilde{c}^{4}+\frac{e \hbar \tilde{c}^{2} B}{2}(4 n-2+\sigma)+\tilde{c}^{2} p_{z}^{2}}
$$

Here, the LL index $n$ runs over positive integers $n=1,2, \ldots$ for the states in the electron and light-hole bands $(\xi= \pm 1)$. For the zero energy, flat heavy-hole band $(\xi=0)$, $n$ runs over all non-negative integers, except 1: $n=0,2,3 \ldots$ The quantum number $\sigma$ accounts for the Kramer's degeneracy lifted by the magnetic field $\mathbf{B}=(0,0, B)$. The corresponding splitting can be viewed as the Zeeman (spin) splitting of LLs: $E_{\xi, n, \uparrow}\left(p_{z}\right)-E_{\xi, n, \downarrow}\left(p_{z}\right)$. The non-parabolicity of the bands implies a strong dependence of this spin-splitting on $p_{z}, B$ and on LL and band indices.

Temperature-induced bandgap opening in narrow gap HgCdTe. Besides the linear behaviour of the absorption coefficient at zero magnetic field measured in both samples (see Supplementary Fig. 1 and Supplementary Note 2), the presence of pseudo-relativistic $3 \mathrm{D}$
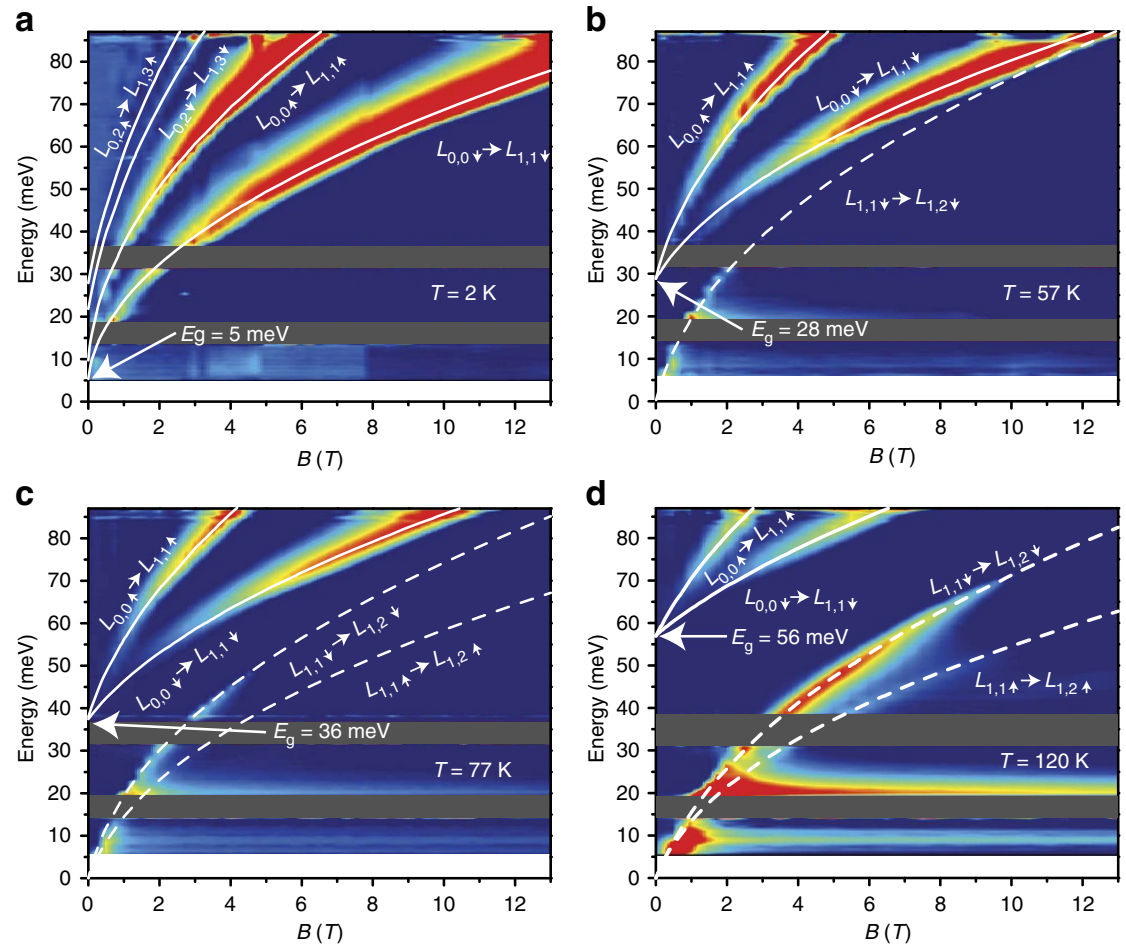

Figure 2 | Relative change of absorbance with magnetic field in sample A. False colour maps present inter-LL transitions as a function of magnetic field. White lines represent the fits using the simplified Kane model, allowing for determination of $\tilde{m}$ and $\tilde{c}$. Solid lines correspond to inter-band transitions, whereas dashed lines account for intra-band transitions. The bandgap values at different temperatures, determined by the inter-band transition energies at zero magnetic field, are depicted with arrows. Shaded areas represent the Reststrahlen bands (between $15-20 \mathrm{meV}$ for $\mathrm{HgTe} / \mathrm{Cd}_{x} \mathrm{Hg}{ }_{1-x} \mathrm{Te} \mathrm{band}_{\mathrm{f}} \mathrm{and}$ 32-37 meV for GaAs substrate band). (a) At $2 \mathrm{~K}$, only inter-band transitions are seen and a $\sqrt{B}$-like behaviour of inter-LL resonances and spin splitting of LLs is observed. (b) At $57 \mathrm{~K}$, intra-band transitions become visible in addition to previous lines. (c,d) At higher temperatures the energy difference between inter-band and intra-band lines at zero field clearly increases, corresponding to a gap opening as a function of temperature. The horizontal line observed at $21 \mathrm{meV}$ in $\mathbf{c}, \mathbf{d}$ corresponds to the energy of TO CdTe-like phonons, arising in magnetoabsorption due to the electron-phonon interaction. As discussed in refs 27,28 , the frequency of such phonon mode in $\mathrm{HgCdTe}$ alloys is almost independent on temperature. In addition, the horizontal line observed at 9 meV in c,d is attributed to optical transitions from impurities as discussed in refs 29,30 . Indeed, because of weak $\mathrm{Hg}$-Te bonds, mercury vacancies are always present in $\mathrm{HgCdTe}$ alloys, even in high-quality $n$-type materials. The amplitude of these lines rises as temperature increases. It has to be noted that thermal energy at $120 \mathrm{~K}$ is $10 \mathrm{meV}$; thus, any features visible below that energy should not be considered as relevant. 
fermions with $\tilde{m} \approx 0$ is established at $2 \mathrm{~K}$ in the sample A by $\sqrt{B}$-like dependence of optical transitions and a spin splitting of LLs seen in Fig. 2a. The fitting of the two main lines based on equation (2) shows that they are related to the inter-band transitions between the heavy-hole band remaining at zero energy $(\xi=0)$ and the $n=1$ spin-up and spin-down LLs with $\xi=1$ (for details, see Supplementary Figs 2 and 3). The extracted bandgap value at $2 \mathrm{~K}$ equals $2 \tilde{m} \tilde{c}^{2}=(5 \pm 2) \mathrm{meV}$. Magneto-optical results obtained at temperatures from 57 to $120 \mathrm{~K}$ are shown in Fig. 2b-d. The bandgap, visualized by intersect of the inter-band transitions with the energy axis (shown by white arrows), increases with temperature (see also Supplementary Fig. 4). In addition to inter-band transitions $(\Delta \xi=1)$, intra-band LLs transitions $(\Delta \xi=0)$ are also observed and fitted with equation (2).

Temperature-driven phase transition. Magneto-absorption of the sample B at different temperatures is presented in Fig. 3, in a $\sqrt{B}$-scale for the sake of clarity. It is seen that at low temperatures and low magnetic field values, the LLs transitions exhibit some discrepancies compared with a pure $\sqrt{B}$ behaviour. Indeed, as shown in Fig. 1, the linear energy dispersion in conduction and valence bands arises in gapless samples only. However, even in the case of small negative (or positive) gap, the bands could be considered as parabolic in the vicinity of the $\Gamma$ point. It corresponds to the small values of parameter $p / \tilde{m} \tilde{c}$, for non-zero rest mass values. At low magnetic fields, the band parabolicity results in a linear behaviour of the LLs transitions as a function of $B$. Therefore, only a precise $\sqrt{B}$ behaviour down to the lowest applied magnetic fields implies a system with genuine massless particles. At temperatures below $T_{C}$, the deviation from a pure $\sqrt{B}$ behaviour is well reproduced by the theory and gives absolute values of the rest mass $\tilde{m}$ approaching to zero when temperature increases. As seen in equation (2), linear extrapolation of optical transitions in the square-root scale intersects the energy axis at $E=E_{\mathrm{g}} / 2$, as represented by arrows in Fig. 3a,b. An accurate $\sqrt{B}$ behaviour for all optical transitions is obtained as an evident proof of gap closing at $77 \mathrm{~K}$ (Fig. 3c, see also Supplementary Fig. 5). Above the critical temperature, the difference in energies of inter- and intra-band transitions in low magnetic fields becomes visible, as it is for the sample A, meaning that a positive gap between the $\Gamma_{6}$ and $\Gamma_{8}$ bands opens. This allows us claiming that at $T_{\mathrm{C}}=77 \mathrm{~K}$, a temperature-driven topological phase transition with pseudo-relativistic massless Kane fermions occurs. This fact is highlighted by the change of a sign of particle rest mass seen in Fig. 4a.

Universal velocity and rest mass description. The rest mass extracted from magneto-optical data and its dependence on temperature is compared with theoretical values using Supplementary equation $(1)^{21}$ (in Supplementary Note 1). In the sample A, the rest mass is positive and increases in the whole range of temperatures, as it is shown in Fig. 4a. As discussed above, in the sample B the Kane fermion rest mass experiences a change of sign corresponding to the temperature-induced semimetal-to-semiconductor topological phase transition, which occurs at $77 \mathrm{~K}$. Supplementary equation (1) ${ }^{21}$ (in Supplementary Note 1) describes very well the experimental rest mass curves for both samples and clearly reproduces the phase transition at $77 \mathrm{~K}$
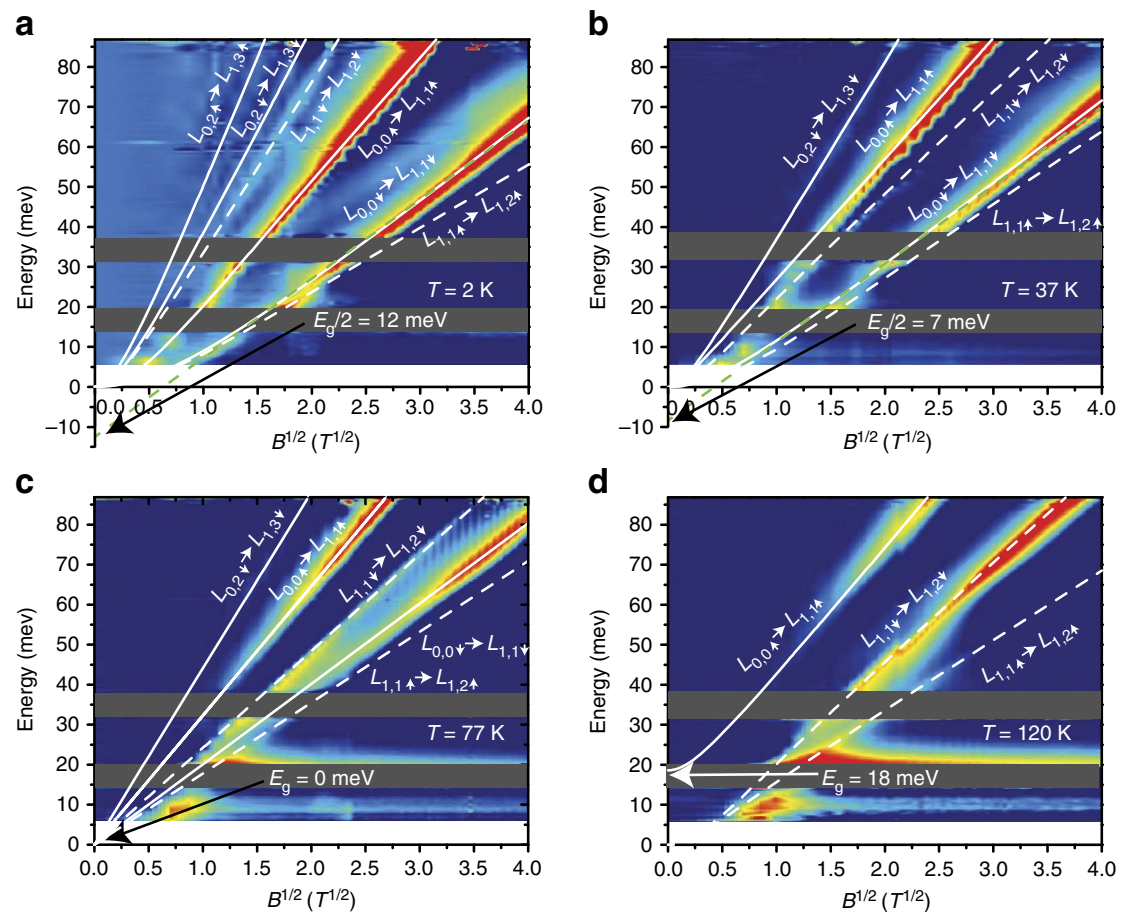

Figure 3 | Relative change of absorbance with magnetic field for sample B. Colour maps of the inter-LL transitions as a function of magnetic field in the sample B at temperatures from 2 to $120 \mathrm{~K}$ in a square root scale. White lines represent the fits using the simplified Kane model. Solid lines represent inter-band optical transitions, whereas dashed lines correspond to intra-band transitions. Green dotted lines are guides for the eyes accounting for half the bandgap energy at each temperature (with arrows indicating half the bandgap values). (a,b) At 2 and $37 \mathrm{~K}$, some discrepancies to the $\sqrt{B}$ behaviour of inter-LL optical transitions are observed, corresponding to the existence of a negative bandgap. (c) At $77 \mathrm{~K}$, a pure $\sqrt{B}$ behaviour corresponds to the gapless state and the presence of genuine massless Kane fermions. (d) At $120 \mathrm{~K}$, a positive gap opens as seen with the presence of an inter-band optical transition. Shaded areas denote the Reststrahlen bands (between 15-20 meV for $\mathrm{HgTe} / \mathrm{Cd}_{x} \mathrm{Hg}_{1-x} \mathrm{Te}$ band and 32-37 meV 150 for GaAs substrate band). The horizontal line at $21 \mathrm{meV}$ in $\mathbf{c}, \mathbf{d}$ is related to the energy of TO CdTe-like phonons, arising in magnetoabsorbance due to the electron-phonon interaction. The line at $9 \mathrm{meV}$ is attributed to optical transitions from impurities. 

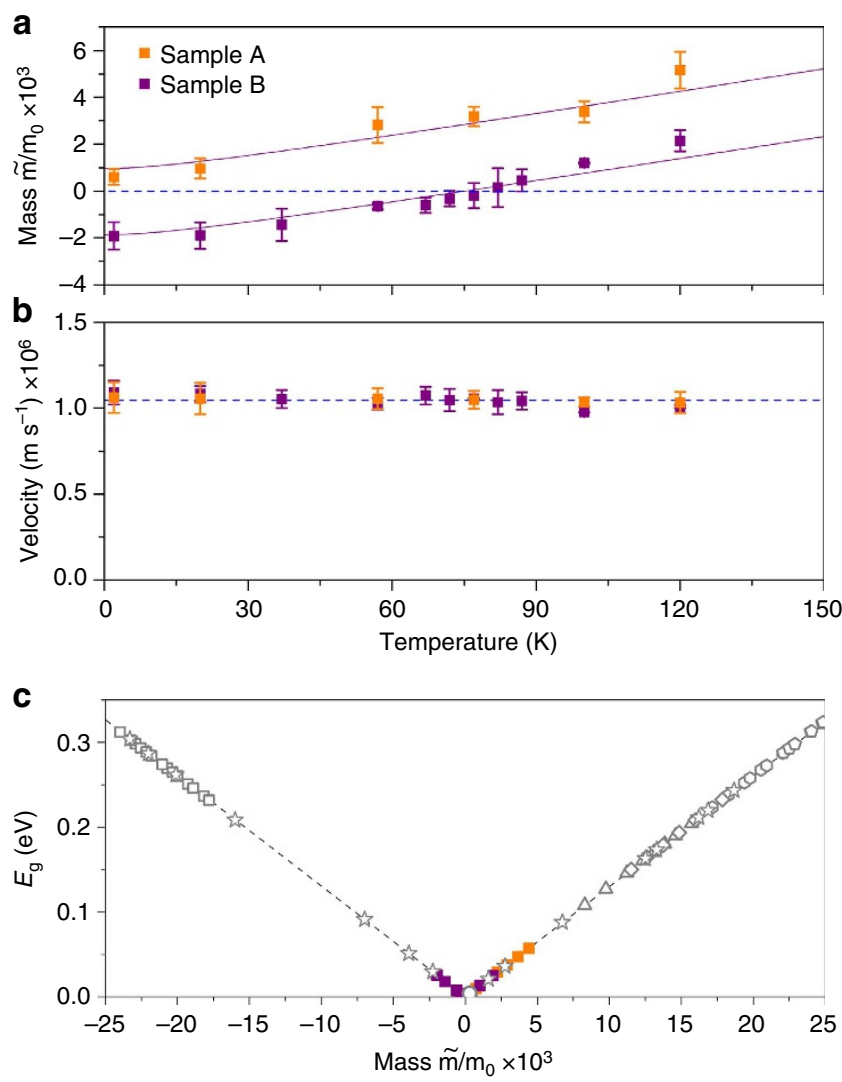

Figure 4 | Kane fermion parameters. Orange and purple points correspond to experimental results obtained in this work, whereas open symbols are experimental points from refs $21,23,24$. (a) Rest mass values for both samples are determined by fitting magneto-optical data with the simplified model, including the $\Gamma_{6}$ and $\Gamma_{8}$ bands only. Kane fermion rest mass in the sample $A$ is close to zero at low temperatures and increases with temperature up to $120 \mathrm{~K}$. In the sample $B$, the Kane particle rest mass is negative at low temperatures, it vanishes at $77 \mathrm{~K}$ and then becomes positive above $T_{\mathrm{c}}$. The lines are theoretical curves calculated using Supplementary equation (1) ${ }^{21}$ (in Supplementary Note 1). (b) The velocity is nearly constant in both samples between 2 and $120 \mathrm{~K}$. The blue dashed line represents the theoretical value. (c) The universal evolution of the bandgap energy versus the Kane fermion rest mass in $\mathrm{HgCdTe}$ alloys is shown. The black dashed line serves as a guide for the eyes. The error bars were estimated by calculating the s.d. of the set of parameters obtained from all fittings at given temperature.

in the sample B. The conic dispersion relation of the massless particles can be therefore realized for the specific range of crystal chemical composition and an according temperature-conditions that provide that the bulk bandgap is fully closed. Interestingly, the Kane fermion velocity $\tilde{c}$ is nearly constant over the whole range of temperatures for both samples with Cd contents of 0.155 and 0.175 . The extracted value of $\tilde{c}=(1.07 \pm 0.05) \times 10^{6} \mathrm{~ms}^{-1}$ is in a very good agreement with the theoretical value defined by $\tilde{c}=\sqrt{2 P^{2} /\left(3 \hbar^{2}\right)}$, which equals to $1.05 \times 10^{6} \mathrm{~ms}^{-1}$ for the wellaccepted value of $E_{\mathrm{P}}=2 m_{0} P^{2} / \hbar^{2} \approx 18.8 \mathrm{eV}$ (ref. 22). Therefore, this universal value of $\tilde{c}$ allows for determination of the particle rest mass for bandgap values in the vicinity of the semimetal-tosemiconductor phase transition induced by temperature, Cd content or other external parameter (for example, pressure). Figure $4 \mathrm{c}$ shows the variation of the experimental bandgap energy obtained in this work (full blue and red points) and from previous studies $^{21,23,24}$ (open symbols), as a function of the rest mass, using $E_{\mathrm{g}}=2 \tilde{m} \tilde{c}^{2}$ and the universal value of $\tilde{c}$.

\section{Discussion}

There are two points limiting the applicability of the simplified Kane model, considering the $\Gamma_{6}$ and $\Gamma_{8}$ band only, for actual $\mathrm{HgCdTe}$ crystals. The first one, already mentioned above, is related to the existence of other bands, considered as remote and not included in the model. The energy gap between the second and the lowest conduction bands in CdTe exceeds $4 \mathrm{eV}$, while the corresponding gap in $\mathrm{HgTe}$ is about $3 \mathrm{eV}$ (ref. 25). Therefore, the cutoff energies for conduction bands in the simplified model should be lower than $3 \mathrm{eV}$. For the valence band, the cutoff energy is defined by the energy difference $\Delta \approx 1 \mathrm{eV}$ between the split-off $\Gamma_{7}$ band and the heavy-hole band. The second limitation is attributed to the flat heavy-hole band, characterized by an infinite effective mass in the model. To ignore the parabolic terms in the electron dispersion of the heavy-hole band, one has to consider sufficiently low energies $E$, such that the relativistic mass of the fermions $E / \tilde{c}^{2}$ should be significantly lower than the heavy-hole mass $m_{\mathrm{hh}}$. Assuming $m_{\mathrm{hh}} \approx 0.5 m_{0}$, where $m_{0}$ is the free electron mass, we arrive at a cutoff energy of $\sim 3 \mathrm{eV}$ for the flat band approximation, which exceeds $\Delta$.

By using temperature as a fine-tuning external parameter we measured the bandgap energy of HgCdTe bulk crystals with wellchosen chemical composition in the vicinity of the semimetal-tosemiconductor phase transition. We clearly observed and accurately measured increasing of the bandgap with temperature in sample A. We also observed genuine massless Kane fermions at the critical temperature of $77 \mathrm{~K}$ in sample B. We used the simplified Kane model to determine the pseudo-relativistic Kane fermion parameters $\tilde{m}$ and $\tilde{c}$ as a function of temperature and Cd content. We observed a change of sign of $\tilde{m}$ accounting for the temperaturedriven topological phase transition. Our results also reveal universal velocity in $\mathrm{HgCdTe}$ crystals allowing for the determination of the Kane fermion rest mass from all experimental results in the literature obtained in the vicinity of the phase transition.

\section{Methods}

Experimental details. We performed magneto-optical studies on two [013]oriented $\mathrm{Hg}_{1-x} \mathrm{Cd}_{x}$ Te layers, with different $\mathrm{Cd}$ concentrations, $x=0.17$ and 0.155 . Both films were sufficiently thick $(\approx 3.2 \mu \mathrm{m})$ to be considered as 3D materials and thin enough to be transparent in the far-infrared spectral range. The samples were grown by molecular beam epitaxy on semi-insulating GaAs substrates with relaxed CdTe buffers ${ }^{26}$. We used a special ultra-high vacuum multi-chamber molecular beam epitaxy set, which allows for the growth of very high-quality HgCdTe crystals monitored by in situ reflection high-energy electron diffraction and single wavelength ultra-fast ellipsometry $(0.5 \mathrm{~nm})$.

The magneto-optical transmission measurements were carried out by using a Fourier transform spectrometer coupled to a $16 \mathrm{~T}$ superconducting coil. The radiation of a Globar lamp is guided to a sample using oversized waveguides (light pipes). The intensity of the transmitted light is measured by a silicon bolometer. Both the magnet and the bolometer require cryogenic temperatures; therefore, most of reported up to today experiments were conducted at $4.2 \mathrm{~K}$ or lower temperatures. In this work, to perform a temperature tuning of the band structure, the standard magneto-optical configuration required important modifications. The bolometer was placed in a vacuum chamber separated from the sample chamber. To provide a wide spectral range for experiments, an indium-sealed cold diamond window ensures the optical coupling between the transmitted light and the bolometer. An additional superconducting coil around the bolometer compensates the spread field of the main coil, keeping the bolometer at zero magnetic field. This additional compensating superconducting coil also provides an additional screening of the bolometer. A Lambda plate coil-placed below the main magnet allows to obtain superfluid helium around the bolometer and keep the main coil at $4 \mathrm{~K}$. Therefore, this modified experimental set-up allows to keep the coils in their superconducting state, the bolometer at its optimal temperature and to tune the sample chamber temperature in the $2-150 \mathrm{~K}$ range.

The magneto-optical spectra were measured in the Faraday configuration up to $16 \mathrm{~T}$, with a spectral resolution of $0.5 \mathrm{meV}$. All the spectra were normalized by the sample transmission response at $B=0 \mathrm{~T}$.

Simplified Kane model. Relativistic fermions are usually described by the Dirac equation:

$$
i \hbar \frac{\partial \Psi}{\partial t}=\left(\beta m c^{2}+c \alpha_{x} p_{x}+c \alpha_{y} p_{y}+c \alpha_{z} p_{z}\right) \Psi
$$


where $p_{i}(i=x, y, z)$ are the components of momentum operator, $m$ and $c$ are the rest mass and velocity of light, respectively. The matrices $\alpha_{i}$ and $\beta$ define the symmetry properties of the particles and have the form

$$
\beta=\left(\begin{array}{cc}
I & 0 \\
0 & -I
\end{array}\right), \quad \alpha_{i}=\left(\begin{array}{cc}
0 & \sigma_{i} \\
\sigma_{i} & 0
\end{array}\right),
$$

in which $\sigma_{i}$ are the Pauli matrices and $I$ is a $2 \times 2$ unit matrix. As it is clearly seen from equation (3), if the rest mass of particles equals zero, their dispersion is described by twofold degenerate cone in energy-momentum space.

Current physics has proven the existence of several bulk condensed-matter materials, which are fairly well described by the above equation. At the same time, there are also other systems with relativistic-like charge carriers; nevertheless, they are described by different Hamiltonians. For Kane fermions, the corresponding Hamiltonian $\hat{H}$ formally resembles the one for genuine 3D Dirac particles. However, we see that it has a form of a $6 \times 6$ matrix, which describes qualitatively a different system:

$$
\hat{H}=\tilde{\beta} \tilde{m} \tilde{c}^{2}+\tilde{c} \tilde{\alpha}_{x} p_{x}+\tilde{c} \tilde{\alpha}_{y} p_{y}+\tilde{c} \tilde{c}_{z} p_{z},
$$

where $\tilde{\beta}=\left(\begin{array}{cc}U_{c} & 0 \\ 0 & U_{c}\end{array}\right), \tilde{\alpha}_{x}=\left(\begin{array}{cc}J_{x} & 0 \\ 0_{i} & -J_{x}\end{array}\right), \tilde{\alpha}_{y}=\left(\begin{array}{cc}J_{y} & 0 \\ 0 & J_{y}\end{array}\right), \tilde{\alpha}_{z}=\left(\begin{array}{cc}0 & J_{z} \\ J_{z} & 0\end{array}\right)$ and where $U_{\mathcal{c}} J_{x}, J_{y}, J_{z}$ are $3 \times 3$ matrices described as follows:

$$
U_{c}=\left(\begin{array}{ccc}
1 & 0 & 0 \\
0 & -1 & 0 \\
0 & 0 & -1
\end{array}\right), \quad J_{x}=\left(\begin{array}{ccc}
0 & \frac{\sqrt{3}}{2} & -\frac{1}{2} \\
\frac{\sqrt{3}}{2} & 0 & 0 \\
-\frac{1}{2} & 0 & 0
\end{array}\right), \quad I_{y}=\left(\begin{array}{ccc}
0 & \frac{i \sqrt{3}}{2} & \frac{i}{2} \\
\frac{i \sqrt{3}}{i} & 0 & 0 \\
\frac{i}{2} & 0 & 0
\end{array}\right), \quad I_{y}=\left(\begin{array}{ccc}
0 & 0 & -1 \\
0 & 0 & 0 \\
-1 & 0 & 0
\end{array}\right)
$$

Here we deliberately present low-energy Hamiltonian, which describes the Kane fermions in a form similar to the Dirac equation, defining the rest mass $\tilde{m}$ and velocity $\tilde{c}$ of the Kane fermions. The matrices $J_{x}, J_{y} J_{z}$ arising in equation (5) do not satisfy the algebra of angular momentum 1; therefore, the Hamiltonian $\hat{H}$ does not reduce to any well-known case of relativistic particles. However, the Kane fermions, with the Hamiltonian described by equation (5), share a number of properties with other relativistic particles.

Data availability. The data that support the findings of this study are available from the corresponding authors on request.

\section{References}

1. Novoselov, K. S. et al. Two-dimensional gas of massless Dirac fermions in graphene. Nature 438, 197-200 (2005).

2. Hasan, M. \& Kane, C. L. Colloquium: topological insulators. Rev. Mod. Phys. 82, 3045-3067 (2010)

3. Qi, X. L. \& Zhang, S.-C. Topological insulators and superconductors. Rev. Mod. Phys. 83, 1057-1110 (2011).

4. Dziawa, P. et al. Topological crystalline states in $\mathrm{Pb}_{1-x} \mathrm{Sn}_{x} \mathrm{Se}$. Nat. Mater. 11, 1023-1027 (2012).

5. Liu, Z. K. et al. A stable three-dimensional topological Dirac semimetal $\mathrm{Cd}_{3} \mathrm{As}_{2}$. Nat. Mater. 13, 677-681 (2014).

6. Lv, B. Q. et al. Observation of Weyl nodes in TaAs. Nat. Phys. 11, 724-727 (2015).

7. Yang, L. X. et al. Weyl semimetal phase in the non-centrosymmetric compound TaAs. Nat. Phys. 11, 728-732 (2015).

8. Orlita, M. et al. Observation of three-dimensional massless Kane fermions in a zinc-blende crystal. Nat. Phys. 10, 233-238 (2014).

9. Malcolm, J. D. \& Nicol, E. J. Magneto-optics of massless Kane fermions: role of the flat band and unusual Berry phase. Phys. Rev. B 92, 035118 (2015).

10. Martyniuk, M., Dell, J. W. \& Faraone, L. in Mercury Cadmium Telluride: Growth, Properties and Applications (eds P. Capper \& J. W. Garland) 153-204 (John Wiley \& Sons, Chichester, 2010).

11. Kane, E. O. in Narrow Gap Semiconductors Physics and Applications: Proceedings of the International Summer School Held in Nimes, France. 3-15 September 1979 (ed. W. Zawadzki). Lecture Notes Phys. 133, 13-31 (1980).

12. Kane, E. O. Band structure of narrow gap semiconductors. Narrow Gap Semicond. Phys. Appl. 133, 13-31 (1980).

13. Bernevig, B. A., Hughes, T. L. \& Zhang, S. C. Quantum spin Hall effect and topological phase transition in HgTe quantum wells. Science 314, 1757-1761 (2006).

14. Jiang, Z. et al. Infrared spectroscopy of Landau levels of graphene. Phys. Rev. Lett. 98, 197403 (2007).

15. Henriksen, E. A. et al. Interaction-induced shift of the cyclotron resonance of graphene using infrared spectroscopy. Phys. Rev. Lett. 104, 067404 (2010).

16. Sadowski, M. L., Martinez, G., Potemski, M., Berger, C. \& de Heer, W. A. Landau level spectroscopy of ultrathin graphite layers. Phys. Rev. Lett. 97, 266405 (2006)

17. Chen, Z.-G. et al. Observation of an intrinsic bandgap and Landau level renormalization in graphene/boron-nitride heterostructures. Nat. Commun. 5, 4461 (2014).

18. Ludwig, J. et al. Cyclotron resonance of single-valley Dirac fermions in nearly gapless HgTe quantum wells. Phys. Rev. B 89, 241406 (R) (2014).
19. Orlita, M. et al. Magneto-optics of massive Dirac fermions in bulk $\mathrm{Bi}_{2} \mathrm{Se}_{3}$. Phys. Rev. Lett. 114, 186401 (2015).

20. Chen, R. Y. et al. Magnetoinfrared spectroscopy of Landau levels and Zeeman splitting of three-dimensional massless Dirac fermions in $\mathrm{ZrTe}_{5}$. Phys. Rev. Lett. 115, 176404 (2015).

21. Laurenti, J. P. et al. Temperature dependence of the fundamental absorption edge of mercury cadmium telluride. J. Appl. Phys. 67, 6454-6460 (1990).

22. Novik, E. G. et al. Band structure of semimagnetic $\mathrm{Hg}_{1-y} \mathrm{Mn}_{y} \mathrm{Te}$ quantum wells. Phys. Rev. B 72, 035321 (2005).

23. Rigaux, C. Interband magnetooptics in narrow gap semiconductors. Narrow Gap Semicond. Phys. Appl. 133, 110-124 (1980).

24. Dornhaus, R. \& Nimtz, G. in The Properties and Applications of the $\mathrm{Hg}_{1-x} \mathrm{Cd} d_{x} \mathrm{Te}$ Alloy System vol. 98 (ed. Gerhard Höhler) 119-281 (Springer Tracts in Modern Physics, 1983).

25. Chadov, S. et al. Tunable multifunctional topological insulators in ternary Heusler compounds. Nat. Mater. 9, 541-545 (2010).

26. Dvoretsky, S. et al. Growth of HgTe quantum wells for IR to THz detectors. J. Electron. Mater. 39, 918-923 (2010).

27. Sheregii, E. M., Cebulski, J., Marcelli, A. \& Piccinini, M. Temperature dependence discontinuity of the phonon mode frequencies caused by a zero-gap state in HgCdTe alloys. Phys. Rev. Lett. 102, 045504 (2009).

28. Polit, J. et al. Additional phonon modes related to intrinsic defects in CdHgTe. Phys. Stat. Solid. C 6, 2012-2015 (2009).

29. Rumyantsev, V. V. et al. Spectra and kinetics of $\mathrm{THz}$ photoconductivity in narrow-gap $\mathrm{Hg}_{1-x} \mathrm{Cd}_{x} \mathrm{Te}(x<0.2)$ epitaxial films. Semicond. Sci. Technol. 28, 125007 (2013).

30. Guldner, Y., Rigaux, C., Mycielski, A. \& Couder, Y. Magnetooptical investigation of $\mathrm{Hg}_{1-x} \mathrm{Cd}_{x}$ Te. Phys. Stat. Sol. (b) 81, 615-627 (1977).

\section{Acknowledgements}

We acknowledge M. Dyakonov, W. Zawadzki and A. Raymond for helpful discussions. This work was supported by the CNRS through LIA TeraMIR project, by the Languedoc-Roussillon region and the MIPS department of Montpellier University via the Terahertz platform, by European cooperation through the COST action MP1204, and the Era.Net-Rus Plus project 'Terasens'. F.T. and S.S.K. acknowledge the support from the Physics Department (INP) of CNRS for post-doc prolongation program. This work was also supported by the Russian Academy of Sciences, the non-profit Dynasty foundation, the Russian Foundation for Basic Research (Grants 14-02-31588, 15-52-16017, 15-02-08274 and 16-02-00672), by Russian Ministry of Education and Science (grant numbers MK-6830.2015.2 and HIII-1214.2014.2) and by NTSIL. J.L., S.M., Z.J. and D.S. acknowledge the support from the U.S. Department of Energy (gran number DE-FG02-07ER46451) for infrared spectroscopy measurements at $4.2 \mathrm{~K}$ that were performed at the National High Magnetic Field Laboratory, which is supported by National Science Foundation (NSF) Cooperative Agreement Number DMR-1157490 and the State of Florida.

\section{Author contributions}

The experiment was proposed by F.T. The theory was formulated by S.S.K. The samples were grown by N.N.M. and S.A.D. The samples were characterized by M.O., A.M.K., W.D., D.B., S.V.M., V.I.G., D.S., J.L., S.M. and Z.J. The temperature-dependent magneto-optical experimental setup was built by C.C., S.R., F.T. and W.K. Magneto-optical experiments were performed by M.M., S.R., F.T., D.S. and C.C. All co-authors discussed the data. F.T., D.S. and W.K. wrote the manuscript.

\section{Additional information}

Supplementary Information accompanies this paper at http://www.nature.com/ naturecommunications

Competing financial interests: The authors declare no competing financial interests

Reprints and permission information is available online at http://npg.nature.com/ reprintsandpermissions/

How to cite this article: Teppe, F. et al. Temperature-driven massless Kane fermions in HgCdTe crystals. Nat. Commun. 7:12576 doi: 10.1038/ncomms12576 (2016).

This work is licensed under a Creative Commons Attribution 4.0 International License. The images or other third party material in this article are included in the article's Creative Commons license, unless indicated otherwise in the credit line; if the material is not included under the Creative Commons license, users will need to obtain permission from the license holder to reproduce the material. To view a copy of this license, visit http://creativecommons.org/licenses/by/4.0/

(C) The Author(s) 2016 